\title{
Unseen but present danger: improving the safe prescribing of anti- embolism stockings (AES)
}

\author{
Alison Bradley
}

NHS Tayside

\begin{abstract}
A strong evidence base exists supporting thromboprophylaxis for venous thromboembolism (VTE) in surgical patients. Given the ageing population, obesity epidemic, and rise in type 2 diabetes, VTE and peripheral vascular disease (PAD) are likely to become an escalating problem. PAD is a contraindication to the use of anti-embolism stockings (AES). Half of those patients diagnosed with PAD report no symptoms, potentially underestimating its prevalence. Implementation of guidelines for thromboprophylaxis, including the safe prescribing of $A E S$, is therefore imperative.
\end{abstract}

The aims of this project were to establish whether thromboprophylaxis was being prescribed correctly, and appropriately, to all surgical inpatients. This included documented evidence that peripheral pulses had been examined - and, in the case of diabetic patients, that there was documentation of full peripheral neurovascular examination - before AES were prescribed. Data were collected from case notes of all surgical inpatients. Foundation year 1 doctors (FY1s) completed a questionnaire assessing their knowledge of local guidelines. Teaching sessions and posters summarising local guidelines were delivered to FY1s.

Appropriate pharmacological prescribing improved from $57.69 \%$ to $100 \%$. AES were appropriately prescribed for $65.38 \%$ of patients. Post intervention this increased to $79.17 \%$. $0 \%$ had documented peripheral neurovascular examination. This increased to $50 \%$ post intervention.

\section{Problem}

Despite clear NHS Tayside guidelines on venous thromboembolism (VTE) prophylaxis for surgical patients, anti-embolism stockings (AES) were often being prescribed without documentation of examination of peripheral pulses. This inferred a lack of awareness of contraindications to AES among junior doctors. Juxtapose this with the known rise in obesity, type 2 diabetes mellitus, and peripheral arterial disease (PAD) - in addition to the fact that over half those diagnosed with PAD report no symptoms (1) - and the potential risk to patient safety posed by inadequate patient assessment becomes apparent. Although prompts to prescribe AES and dalteparin existed on kardexs, there was a lack of prompts and space to document peripheral neurovascular examination on preassessment patient clerk-in documents which compounded the problem further.

\section{Background}

There is a strong evidence base supporting thromboprophylaxis for VTE in surgical patients. Increased age is a known risk factor for VTE. In light of the ever increasing elderly population, VTE is likely to become an increasing problem. Furthermore, 2.7 million people in the UK aged over 55 years have peripheral arterial disease (PAD), the incidence increasing to $15-20 \%$ of the population aged over 70 years $(1,2)$. Fifty per cent of those individuals diagnosed with PAD report no symptoms $(1,2)$. Therefore, when considering what is known about increasing rates of known risk factors for PAD, including increased age, obesity, and diabetes, the potential for underestimating the prevalence of PAD becomes apparent. It is therefore imperative that guidelines for thromboprophylaxis, including the safe prescribing of AES, are implemented.

A plethora of studies exist which focus on improving assessment of VTE risk and correct prescribing of VTE prophylaxis. Watts and Grant (3) showed that simple cost effective measures, including teaching sessions and poster reminders, could improve the assessment of VTE risk and prescribing of appropriate VTE prophylaxis in orthopaedic patients. However, like many other studies, this did not focus on the safe prescribing of AES, including junior doctors' knowledge of contraindications to AES.

The aim of the current project was therefore to utilise what had been learned from the cost effective measured employed by Watts and Grant (3) and apply it to improving patient safety in our department by focusing on safe prescribing of AES in addition to VTE risk assessment and prophylaxis.

\section{Baseline Measurement}

Before initiating this project, data were collected from all surgical inpatient notes and kardexs to ascertain the scale of the problem. Information collected included whether patients were elective or emergency admissions. Kardexs were examined to explore whether dalteparin (Fragmin) was prescribed and, if so, at what dose. If dalteparin was not prescribed, medical and nursing notes were examined to see whether there was a documented reason why it was not prescribed. Data were also collected on whether AES were prescribed and if they were being worn by the patients. If AES were 
not prescribed, nursing and medical notes were examined to ascertain a reason for this. The patients' past medical histories were examined to ascertain whether they had any known contraindication to AES. If patients had diabetes, data were collected on whether a peripheral neurovascular examination was documented. All patient notes were audited for the presence of a documented examination of peripheral pulses before prescribing AES.

Appropriate prescribing of pharmacological VTE prophylaxis was evident in $57.69 \%$ of patients. AES were appropriately prescribed for $65.38 \% .0 \%$ had documented peripheral neurovascular examination. Three patients had type 2 diabetes mellitus, and none of these had a documented peripheral neurovascular examination.

\section{Design}

When considering the cause of this problem it became apparent that the local VTE prophylaxis guidelines were very clear and easy to read. However, awareness of these guidelines and knowledge on how to access them were lacking among junior doctors. Previous successful departmental projects including prompts on preprinted kardexs to prescribe dalteparin and AES, while successful in emphasising the importance of VTE prophylaxis, overshadowed conveying the message to foundation year 1 doctors (FY1s) about the importance of knowing contraindications to AES.

Following discussion with the multidisciplinary team it became clear that knowledge of the guidelines, including safe prescribing of AES, must be improved. Qualitative feedback was gathered from the FY1s on the best ways to achieve this. It was agreed that teaching sessions and posters summarising the guidelines were popular, cost effective and sustainable options. Furthermore, FY1s raised the fact that pre-assessment medical documents for elective patients lacked both prompts and space to document peripheral neurovascular examination. Patients who were not pre-assessed were clerked on a document which contained both prompts and space for documenting peripheral neurovascular examination. It was therefore decided that all patients should be clerked on this existing document. This was easily implemented due to familiarity with the document as well as being cost effective and sustainable.

\section{Strategy}

PDSA cycle 1: The issue of safe prescribing of AES was discussed at the hospital clinical effectiveness meeting with senior representatives of the multidisciplinary team. During this meeting the results from baseline measurements were presented. It was decided that the issue of safe prescribing of AES should be addressed through teaching sessions for FY1s.

PDSA cycle 2: Before awareness of guidelines among FY1s could be address, it had to first be established whether this was the true cause of the problem. An anonymous questionnaire was created and administered to all FY1s working in the surgical department. It focused on whether the FY1s knew the correct dose of pharmacological VTE prophylaxis as detailed in the local policy, the correct way to prescribe AES, and their ability to list contraindications to AES. Fifty per cent of the FY1s knew the recommended prophylactic dose of dalteparin for surgical patients; $100 \%$ knew that AES had to be prescribed; $25 \%$ did not know any contraindications to AES; $25 \%$ could name one contraindication; and $50 \%$ could name three contraindications.

PDSA cycle 3: A session was held with the FY1s to discuss the findings of the questionnaire and to seek qualitative feedback on the existing policy and how practice could be improved. The following issues were raised: need for teaching sessions on the local policy for VTE prophylaxis including contraindications to AES; posters displayed in the doctors' rooms summarising the policy; and the lack of prompts and space on pre-admission paperwork to record peripheral neurovascular examination.

PDSA cycle 4: Teaching sessions outlining the local VTE prophylaxis policy and focusing on contraindications to AES were delivered to FY1s and a copy of the policy circulated via email.

PDSA cycle 5: Teaching sessions were backed by creation of a poster outlining the local VTE prophylaxis policy. These posters were displayed in the doctors' rooms.

PDSA cycle 6: It was decided that all patients, whether elective or emergency admissions, should have a full clerk-in on the preexisting documents, which contain a visual prompt and space to document a peripheral neurovascular examination.

PDSA cycle 7: Data were collected which demonstrated improvement in practice following these interventions. Teaching sessions and posters were therefore included in induction sessions for all new foundation doctors coming to work in the surgical department.

\section{Results}

Appropriate pharmacological prescribing improved from $57.69 \%$ to $100 \%$. AES were appropriately prescribed for $65.38 \%$ preintervention. Post intervention this increased to $79.17 \%$. AES were worn by $34.78 \%$ and this increased slightly post intervention to $36.84 \%$. No patients $(0 \%)$ had documented peripheral neurovascular examination, and this increased to $50 \%$ post intervention. The percentage of diabetic patients who had peripheral neurovascular examination documented increased from $0 \%$ to $33 \%$.

\section{Lessons and Limitations}

This study was limited by small sample size and short study period. However, there was not a high turnover in staff during the study period. FY1s were also not aware that the data were being collected pre- and post intervention to avoid bias.

This project demonstrated a clear improvement in the quality of practice, while being cost effective. In order to sustain and build on improvements demonstrated in this project, teaching sessions and poster summaries of VTE prophylaxis will now be incorporated into 


\section{BMJ Quality Improvement Reports}

induction sessions for new foundation year doctors.

\section{Conclusion}

Improvement was demonstrated in the prescribing of thromboembolism prophylaxis and documentation of peripheral neurovascular examination before prescribing AES. Interventions used to highlight and improve this area of practice were cost effective and will be sustainable by incorporating such teaching sessions into induction of all foundation year doctors rotating through the surgical department. Future audit will focus on improving prescribing compliance with local guidelines, and compliance with prescribing to ensure that improvement is sustained and further built upon.

\section{References}

1. Fowkes FG, Rudan D, Rudan I, et al. Comparison of global estimates of prevalence and risk factors for peripheral artery disease in 2000 and 2010: a systematic review and analysis. Lancet 2013;382:1329-40.

2. Peach G, Griffin M, Jones KG, et al. Diagnosis and management of peripheral arterial disease. BMJ 2012;345:e5208.

3. Watts L, Grant D. Venous thromboembolism (VTE) risk assessment and prophylaxis in acute orthopaedic admissions: improving compliance with national guidelines. BMJ Qual Improv Report 2013;2: doi:10.1136/bmjquality.u202229.w1118

\section{Declaration of interests}

Nothing to declare.

\section{Acknowledgements}

Dr Pei Jin Lim (FY1) 